\title{
Compliance with Tuberculosis Screening in Irregular Immigrants
}

\author{
Francesca Bonvicini ${ }^{1}$, Silvia Cilloni ${ }^{2}$, Rossano Fornaciari ${ }^{1}$, Carmen Casoni ${ }^{1}$, \\ Cristina Marchesi ${ }^{1}$, Marina Greci ${ }^{1}$, Lucia Monici ${ }^{1}$, Fausto Nicolini ${ }^{1}$ and Marco Vinceti ${ }^{2, *(\text { (D) }}$ \\ 1 Azienda Unità Sanitaria Locale di Reggio Emilia, Reggio Emilia 42122, Italy; \\ francesca.bonvicini2@ausl.re.it (F.B.); rossano.fornaciari@ausl.re.it (R.F.); carmen.casoni@ausl.re.it (C.C.); \\ cristina.marchesi@ausl.re.it (C.M.); marina.greci@ausl.re.it (M.G.); lucia.monici@ausl.re.it (L.M.); \\ fausto.nicolini@ausl.re.it (F.N.) \\ 2 Università degli Studi di Modena e Reggio Emilia, Dipartimento di Scienze Biomediche, \\ Metaboliche e Neuroscienze, Modena 41125, Italy; silvia.cilloni@unimore.it \\ * Correspondence: marco.vinceti@unimore.it; Tel.: +39-059-205-5481
}

Received: 23 November 2018; Accepted: 21 December 2018; Published: 23 December 2018

\begin{abstract}
Tuberculosis (TB) is a serious public health problem in many regions of the world, especially in the poorest areas. For this reason, screening for active and latent forms must be considered when dealing with high-risk groups such as irregular immigrants in Western countries. We conducted a retrospective cohort study by recruiting subjects aged $\geq 15$ years who underwent a tuberculin skin test at a dedicated National Health Service Centre in a northern Italian province between 1 January 2012 and 31 December 2013. These participants were followed up until 31 December 2016. We aimed at evaluating an experimental protocol for active and latent tuberculosis screening, focusing on patient compliance, feasibility, and capability to detect clinical forms of the disease. We enrolled 368 irregular immigrants, i.e., immigrants not having a valid residence permit and who were therefore not entitled to choose a general practitioner. In total, $90.22 \%$ of these completed all the steps for the screening of active TB, while $87.33 \%$ also undertook screening for the latent form of the disease. Homelessness, self-reported prostitution, female sex, and employment status adversely affected compliance. Chronic alcohol consumption was associated with increased risk of no beginning or interruption of the procedures. All of the five patients with active TB successfully completed the treatment. Overall, adherence to the screening program was high compared to other studies in immigrants, possibly owing to organizational factors such as the availability of cultural mediators, the network between the different health services, the presence of dedicated nursing staff and a free-of-charge service. In addition, selected vulnerable subgroups should be targeted using tailored screening and follow-up programs.
\end{abstract}

Keywords: tuberculosis; screening for tuberculosis; public health; immigrants

\section{Introduction}

Tuberculosis (TB) is the main cause of death from infectious disease globally. Drug-resistant forms of the disease are a major risk to global health security [1]. The WHO estimates that 10.4 million individuals became ill with TB and 1.7 million died in 2016. Despite a fall in mortality rate by $3 \%$ per year, TB remains the ninth leading cause of death worldwide, resulting in 1.3 million deaths in HIV-negative people and almost 400,000 deaths in HIV-positive people [2,3]. TB epidemiology in most low-incidence countries is characterized by a low rate of transmission in the general population, occasional outbreaks and a majority of TB cases generated from progression of latent tuberculosis infection (LTBI) rather than local transmission [4]. Migration flows have changed drastically since the 
beginning of the 21st century. Because most immigrants come from countries with a high incidence of tuberculosis, the contribution of the migrant population to new cases of tuberculosis is stronger in relative terms than for its weight in the total population [5]. For this reason, it is necessary to both diagnose TB early by including universal drug susceptibility testing, and to implement systematic screening for TB in selected high-risk groups. Early detection helps to reduce the risk of further TB transmission, poor treatment outcomes and undesirable health sequelae, thus also decreasing adverse social and economic consequences of the disease [6,7].

In the last 50 years, the annual incidence of TB in Italy decreased by about $70 \%$, from around 25 to 7 cases per 100,000 individuals, and it now seems to be quite stable [8]. In this country, however, only limited data are available about TB epidemiology in potentially high-risk groups such as undocumented immigrants. Consequently, TB prevalence and risk factors in illegal immigrants are unknown. Furthermore, they have not been monitored over time. In spite of this, while national guidelines [9] recommend LTBI screening in high-risk subjects such as pulmonary TB contacts, HIV-infected patients, and patients undergoing immunosuppressive therapy and health care workers, less attention is devoted to the immigrant population, whereby early detection of active and LTBI cases should be pursued using the following strategies [10]: screening for both active TB and LTBI and therapy of LTBI in children, chest X-ray (CXR) screening for active TB in symptomatic subjects especially recent irregular immigrants. In addition, screening for active and latent $\mathrm{TB}$ in asymptomatic adults with recent immigration ( $<5$ years) or living in social and health conditions of marginalization has no strong evidence. This also applies to undocumented immigrants, although these may represent an important source of LTBI and active TB. At the Centre for Health of Foreign Family (CFF), systematic screening for both active TB and LTBI had been performed since January 2012.

\section{Materials and Methods}

\subsection{Setting and Clinical Procedures}

The study protocol was approved by the Ethics Committee of the Reggio Emilia Province on 19 April 2017 and by the Reggio Emilia Local Health Unit on 5 June 2017 (document code 2017/DS/0038).

This study took place in the CFF of Reggio Emilia, an area in northern Italy with approximately 550,000 inhabitants. The CFF is an outpatient clinic located in the city centre. It is devoted to immigrants without a valid residence permit, who are not entitled to choose a general practitioner.

In accordance with national recommendations [10], the reference test for the diagnosis of LTBI is the tuberculin skin test (TST) based on the Mantoux method. TST is carried out by the nursing staff in accordance with the Mantoux method, by inoculating $0.1 \mathrm{~mL}$ of purified protein [11]. The nursing or medical staff read the induration in millimetres after $48-72 \mathrm{~h}$. If positive, the TST is always evaluated by medical personnel. The cut-off for TST positivity is usually $10 \mathrm{~mm}$. In case of immunosuppression, it drops to $5 \mathrm{~mm}$. Screening is performed according to a local protocol based on the combined use of TST and interferon gamma release assay (IGRA). Specifically we have used Quantiferon-TB Gold (QFT) for evaluating the possible presence of active TB. In Figure 1 it is possible to see the algorithm used at CFF for the screening of active TB and LTBI; in immigrants with any symptom of TB and/or peripheral lymphadenopathy, TST is performed as a first step. As far as TB symptoms are concerned, we consider at least one of the following: cough, haemoptysis, fever, chest pain, weight loss, fatigue, night sweats, chills or loss of appetite. The clinician visiting the patient may prescribe CXR at the time of TST and at any time during the screening protocol if a high risk for active TB is estimated. If the CXR shows any abnormality, the patient is referred for the pneumological examination (PE) or hospitalization. If CXR is negative, the patient's LBTI diagnosis can be proceeded with. TST-positive patients undergo CXR (if not yet performed) and possibly QFT. QFT is generally prescribed for confirmation for the purpose of offering LTBI treatment. The indication for LTBI treatment is decided by the pneumologist, after patient examination and evaluation of CXR and QFT results. For LTBI treatment, we generally prescribe isoniazid for 6 months at a dose of $5 \mathrm{mg} / \mathrm{kg} /$ day, combined with vitamin B6, in order to 
prevent neurological adverse effects. Isoniazid preventive therapy (IPT) is recommended in people aged $\leq 35$ years and in people of any age with HIV co-infection. In case of contraindication, failure to accept or need to discontinue IPT, patients with LTBI undergo health surveillance (HS) through clinical examination every six months for two years, for the purpose of early detection of the onset of active TB symptoms.

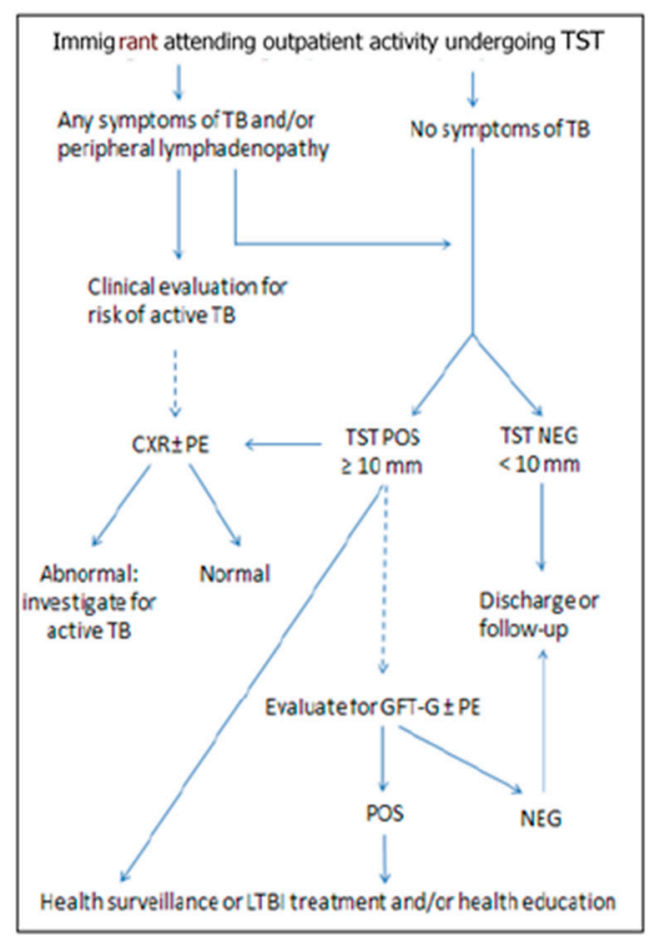

Figure 1. Procedures for active tuberculosis (TB) and latent tuberculosis infection (LTBI) screening at the Centre for Health of Foreign Family (CFF) of Reggio Emilia. TST: Tuberculin Skin Test; CXR: chest $X$-ray; PE: Pneumological Examination; POS: positive; NEG: negative.

All examinations and visits are free of charge. Cultural mediators belonging to the most common ethnic groups of patients are constantly present to assist health personnel and immigrants during outpatient activity.

\subsection{Study Design and Definitions}

In this retrospective cohort study, all foreign-born patients aged $\geq 15$ years who underwent TST at the CFF between 1 January 2012 and 31 December 2013 were enrolled, provided that there was no evidence of previous contact with a case of pulmonary TB or positive TST. The period of active follow-up in patients undergoing HS ended on 31 December 2016. We defined patients with LTBI as immigrants with both positive TST and QFT and patients with positive TST but QFT not requested or not performed. We considered patients without LTBI as subjects with negative TST and with positive TST but negative QFT. According to the World Health Organization [12], a bacteriologically confirmed TB case was one from whom a biological specimen was positive by smear microscopy, culture or rapid diagnostic tests (such as Xpert MTB/RIF assay that is a nucleic acid amplification test). A clinically diagnosed TB case was one diagnosed with active TB by a clinician or other medical practitioner who decided to start a full course of TB treatment; this definition included cases diagnosed on the basis of X-ray abnormalities or suggestive histology and extra pulmonary cases without laboratory confirmation. 


\subsection{Data Analysis}

We analysed compliance with screening for active TB, LTBI, and the whole protocol, also taking patient characteristics into account. We also assessed the capacity of the adopted procedures to detect LTBI and active TB. In order to do this, we computed odds ratios (ORs) along with their 95\% confidence interval (CI) using crude and multivariable logistic regression models. The potential confounders included in multivariable analysis were adjusted for sex, age, presence of cough, education, knowledge of Italian, employment status (employed versus unemployed), years from arrival in Italy and homelessness. We included knowledge of Italian language because it could affect compliance, influencing patient's ability to understand the motivations of the clinical examinations and therapies proposed. When the use of multivariable analysis led to exceedingly high statistical instability, we limited our assessment to crude estimates only (unadjusted for other variables). Risk analysis was performed by calculating odds ratios estimated from conditional logistic regression with crude and multivariate models. When the odds ratios could not be calculated, we applied the chi2 test and probability according to Fisher's exact Test. For the evaluation of the differences between continuous variables, we applied the $t$-test. For PE, HS, and IPT, we performed only the crude analysis because of the smallness of the sample, causing too much instability in the statistical analysis.

\section{Results}

From 2012 to 2016, TST was administered to a total number of 404 immigrants. Of these, 36 patients were excluded from our analysis: 8 were born in Italy from undocumented parents and 28 were younger than 15 years. The final study population consisted of 368 immigrants: 186 (50.5\%) and 182 (49.5\%) were tested in 2012 and 2013, respectively. The demographic characteristics of the study population are reported in Table S1. Adherence to the various stages of the screening procedures and treatment outcomes are shown in Table 1.

Table 1. Compliance with the different steps of the algorithm and treatment outcomes.

\begin{tabular}{|c|c|c|}
\hline Steps of the Algorithm & $N$ Performed/Prescribed & $\%$ \\
\hline TST read & $349 / 368$ & 94.8 \\
\hline CXR performed & $134 / 151$ & 88.7 \\
\hline QFT performed & $89 / 110$ & 80.9 \\
\hline PE attended & $78 / 84$ & 92.9 \\
\hline Screening TB (TST, CXR, PE) completed & $332 / 368$ & 90.2 \\
\hline negative TST with CXR indication & $12 / 12$ & 100 \\
\hline positive TST & $122 / 139$ & 87.8 \\
\hline Screening LTBI (TST, CXR, QFT, PE) completed & $317 / 363$ & 87.3 \\
\hline negative TST with CXR indication & $12 / 12$ & 100 \\
\hline positive TST & $107 / 134$ & 79.9 \\
\hline \multicolumn{3}{|l|}{ LTBI treatment } \\
\hline refusal or voluntary interruption & $8 / 28$ & 28.6 \\
\hline medical contraindication or interruption & $6 / 28$ & 21.4 \\
\hline treatment completed & $14 / 28$ & 50.0 \\
\hline $\begin{array}{c}\text { LTBI health surveillance } \\
\text { never started }\end{array}$ & $17 / 41$ & 41.5 \\
\hline voluntary interruption & $18 / 41$ & 43.9 \\
\hline completed & $6 / 41$ & 14.6 \\
\hline TB treatment completed & $5 / 5$ & 100 \\
\hline Whole algorithm completed & $281 / 368$ & 76.4 \\
\hline negative TST with CXR indication & $12 / 12$ & 100 \\
\hline positive TST & $71 / 139$ & 51.1 \\
\hline
\end{tabular}

TST: tuberculin skin test; CXR: chest-X-ray; QFT: quantiferon test; PE: pneumological examination; TB: tuberculosis; LTBI: latent tuberculosis infection. 
Patients who did not return for the reading of TST amounted to $15.2 \%$. Patients who did not undergo CXR, QFT and PE despite the prescriptions amounted to $11.3 \%, 19.1 \%$ and $7.1 \%$, respectively. Finally, $90.2 \%$ of subjects completed all steps expected for the screening of active TB, considering TST reading and CXR/PE, when prescribed. In immigrants with a positive TST result, adherence was lower, since their path is usually longer. A total of 5 cases of active TB were diagnosed during the study period. After discharge from hospital, they were followed monthly at TBOA (Outpatient Activity dedicated to management of LTBI and TB at CFF) and they all successfully completed the treatment.

The overall compliance with LTBI screening, which involved TST, CXR, QFT, and PE was $87.3 \%$. Also in this case, adherence of patients with positive TST was observed to be lower $(79.9 \%)$. LTBI treatment was prescribed to 28 patients, while only 20 out of 28 patients started prophylaxis with Isoniazid, because eight patients had increased levels of transaminases for chronic alcohol abuse or $\mathrm{HBsAg}$, or they refused therapy. Eventually, 14 of the 20 patients who started IPT were able to complete the treatment, whereas 6 patients discontinued therapy voluntarily or after medical indication. HS for 2 years was indicated in 41 patients yet only 6 completed all follow-up. Finally, 76.4\% of immigrants completed the whole diagnostic and therapeutic protocol for active TB and LTBI. Considering only TST-positive ones, adherence decreased to $51 \%$.

Tables 2 and 3 deal with the full path for the screening of active TB and LTBI. In multivariable analysis regarding the screening of active TB (Table 2), it is possible to appreciate that female sex, homelessness and age between 25-34 years were associated with a considerably lower rate of completion of the screening. On the contrary, a higher level of education, time of arrival in Italy for at least 5 years and the presence of cough were all associated with better compliance. With regard to LTBI (Table 3), being a native of the Americas, age between 25 and 34 years, employment, and homelessness were associated with considerably lower adherence. Refugee status, time of arrival in Italy for at least 5 years and the presence of cough were associated with better compliance. It is interesting to note that being from the Americas was associated with very low compliance for both active TB and LTBI screening (OR 0.27, 95\% CI 0.05-1.44; OR 0.32, 95\% CI 0.06-1.64, respectively). Table 4 outlines compliance with the full algorithm. As can be seen, age had a negative impact on adherence. In addition, being employed, homelessness and prostitution were associated with considerably lower compliance. Being a native of South-East Asia and the Western Pacific (OR 4.48, 95\% CI 1.57-12.73), presence of cough, having been in Italy for a longer time, in particular for more than 5 years, and the attainment of higher education levels were observed to be related to a much higher probability of completion. Female Chinese patients' compliance with the protocol was substantially higher than in the rest of the population (OR 55.63, 95\% CI 5.35-578), while Nigerians, Georgians, and Tunisians had lower levels of adhesion (Table S2).

Considering compliance with TST reading, both bivariate and multivariate analysis show that American and European origin, age between 25-34 years old, prostitution and homelessness were associated with considerably lower rates of return for the reading of the test. On the other hand, higher levels of education were associated with better compliance. In multivariable analysis, the presence of cough was the only symptom associated with greater compliance (OR 7.61, 95\% CI 0.92-63.2) (Table S3). Compliance with CXR showed a positive correlation to the presence in Italy for at least 5 years, while a negative correlation was associated to the female sex (Table S4). Compliance with QFT was inversely correlated with education and positively associated with the age group 25-34 years (OR 3.80, 95\% CI 0.87-16.53) (Table S5). Female sex, being a native of the Americas, and sufficient or good knowledge of Italian were associated with a $100 \%$-rate of IPT completion. Conversely, refugees showed a tendency to refuse or to voluntarily stop the treatment, and higher education levels inversely correlated with the probability of completion (Table S6). 
Table 2. Association between selected characteristics and compliance with screening for active tuberculosis (TB).

\begin{tabular}{|c|c|c|c|c|c|c|}
\hline \multirow[t]{2}{*}{ Sample Characteristics } & \multicolumn{2}{|c|}{$\begin{array}{c}\text { TВ Screening } \\
\text { Started/Completed }\end{array}$} & \multirow{2}{*}{$\begin{array}{l}\text { Bivariate OR } \\
\quad(95 \% \mathrm{CI})\end{array}$} & \multirow[t]{2}{*}{$p$-Value } & \multirow{2}{*}{$\begin{array}{l}\text { Multivariate OR } \\
\quad(95 \% \mathrm{CI})\end{array}$} & \multirow[t]{2}{*}{$p$-Value } \\
\hline & $N$ & $\%$ & & & & \\
\hline \multicolumn{7}{|l|}{ Sex: } \\
\hline Male & $222 / 243$ & 91.36 & 1.00 (reference) & & 1.00 (reference) & \\
\hline Female & $110 / 125$ & 88.00 & $0.69(0.34-1.40)$ & 0.306 & $0.47(0.22-1.03)$ & 0.061 \\
\hline \multicolumn{7}{|l|}{ Age at TST in Years: } \\
\hline $15-24$ & $90 / 97$ & 92.78 & 1.00 (reference) & & 1.00 (reference) & \\
\hline $25-34$ & $113 / 130$ & 86.92 & $0.52(0.21-1.30)$ & 0.161 & $0.44(0.16-1.18)$ & 0.103 \\
\hline $35-44$ & $87 / 95$ & 91.58 & $0.85(0.29-2.43)$ & 0.756 & $0.57(0.18-1.84)$ & 0.344 \\
\hline$\geq 45$ & $42 / 46$ & 91.30 & $0.82(0.23-2.94)$ & 0.757 & $0.90(0.20-4.03)$ & 0.891 \\
\hline Continuous OR & & & $0.99(0.70-1.41)$ & 0.972 & $0.96(0.64-1.43)$ & 0.827 \\
\hline \multicolumn{7}{|l|}{ Region of Origin: } \\
\hline Africa & $103 / 111$ & 92.79 & 1.00 (reference) & & 1.00 (reference) & \\
\hline Eastern Mediterranean & $75 / 83$ & 90.36 & $0.73(0.26-2.03)$ & 0.544 & $0.75(0.25-2.27)$ & 0.607 \\
\hline Europe & $60 / 72$ & 83.33 & $0.39(0.15-1.00)$ & 0.051 & $0.44(0.15-1.30)$ & 0.138 \\
\hline SE Asia/West Pacific & $85 / 90$ & 94.44 & $1.32(0.42-4.19)$ & 0.637 & $2.49(.57-10.87)$ & 0.226 \\
\hline Americas & $9 / 12$ & 75.00 & $0.23(0.05-1.04)$ & 0.056 & $0.27(0.05-1.44)$ & 0.125 \\
\hline TB Incidence $^{\circ}:$ & & & $1.26(0.89-1.79)$ & 0.187 & $1.15(0.79-1.69)$ & 0.458 \\
\hline \multicolumn{7}{|l|}{ Any Symptoms *: } \\
\hline No & $211 / 236$ & 89.41 & 1.00 (reference) & & & \\
\hline Yes & $121 / 132$ & 91.67 & $1.30(0.62-2.74)$ & 0.485 & & \\
\hline \multicolumn{7}{|l|}{ Cough: } \\
\hline No & $249 / 280$ & 88.93 & 1.00 (reference) & & 1.00 (reference) & \\
\hline Yes & $83 / 88$ & 94.32 & $2.07(0.78-5.49)$ & 0.145 & $3.23(1.04-10.05)$ & 0.043 \\
\hline Education **: & & & $1.36(0.88-2.12)$ & 0.168 & $1.49(0.93-2.40)$ & 0.101 \\
\hline Italian Language ${ }^{* * 0}$ : & & & $0.89(0.52-1.50)$ & 0.652 & $0.90(0.51-1.58)$ & 0.721 \\
\hline \multicolumn{7}{|l|}{ Employment Status: } \\
\hline No & $234 / 258$ & 90.70 & 1.00 (reference) & & 1.00 (reference) & \\
\hline Yes & $90 / 102$ & 88.24 & $0.77(0.37-1.60)$ & 0.484 & $0.79(0.32-1.96)$ & 0.612 \\
\hline \multicolumn{7}{|l|}{ Years in Italy: } \\
\hline$<5$ & $223 / 252$ & 88.49 & 1.00 (reference) & & 1.00 (reference) & \\
\hline$\geq 5$ & $102 / 109$ & 93.58 & $1.89(0.80-4.47)$ & 0.144 & $3.29(1.18-9.22)$ & 0.023 \\
\hline Continuous OR & & & $1.06(0.96-1.18)$ & 0.248 & $1.18(0.72-1.92)$ & 0.513 \\
\hline \multicolumn{7}{|l|}{ Homelessness: } \\
\hline No & $313 / 345$ & 90.72 & 1.00 (reference) & & 1.00 (reference) & \\
\hline Yes & $19 / 23$ & 82.61 & $0.49(0.16-1.52)$ & 0.213 & $0.35(0.10-1.25)$ & 0.107 \\
\hline \multicolumn{7}{|l|}{ Refugees: } \\
\hline No & $304 / 340$ & 89.41 & - & & & \\
\hline Yes & $28 / 28$ & 100.00 & - & $0.070^{* * *}$ & & \\
\hline \multicolumn{7}{|l|}{ Prostitution: } \\
\hline No & $323 / 357$ & 90.48 & 1.00 (reference) & & & \\
\hline Yes & $9 / 11$ & 81.82 & $0.47(0.10-2.28)$ & 0.352 & & \\
\hline \multicolumn{7}{|l|}{ Pregnancy: } \\
\hline No & $323 / 359$ & 89.97 & - & & & \\
\hline Yes & $9 / 9$ & 100.00 & - & $0.319^{* * *}$ & & \\
\hline \multicolumn{7}{|l|}{ TST Result: } \\
\hline Negative & $210 / 210$ & 100.00 & - & & & \\
\hline Positive & $122 / 139$ & 87.77 & - & $0.000 * * *$ & & \\
\hline
\end{tabular}

${ }^{\circ}:$ TB incidence in the country of origin is sub-divided into 4 categories: 0-49, 50-99, 100-199, $\geq 200$ per 100,000 population/year. *: Meaning at least one among cough, hemoptysis, fever, chest pain, weight loss, fatigue, night sweats, chills or loss of appetite. ${ }^{* *}$ : Education is sub-divided into three categories: illiterate/primary school, secondary school, high school/degree. ${ }^{* * 0}$ : Knowledge of Italian is sub-divided into three categories: none, sufficient, good. ***: Two-sample Mann-Whitney test. TB: tuberculosis; LTBI: latent tuberculosis infection; TST: tuberculin skin test; SE Asia: South-East Asia; OR: odds ratio; CI: confidence interval. 
Table 3. Association between selected characteristics and compliance with screening for latent TB infection (LTBI).

\begin{tabular}{|c|c|c|c|c|c|c|}
\hline \multirow[t]{2}{*}{ Sample Characteristics } & \multicolumn{2}{|c|}{$\begin{array}{l}\text { LTBI Screening } \\
\text { Started/Completed }\end{array}$} & \multirow{2}{*}{$\begin{array}{l}\text { Bivariate OR } \\
\quad(95 \% \mathrm{CI})\end{array}$} & \multirow[t]{2}{*}{$p$-Value } & \multirow{2}{*}{$\begin{array}{c}\text { Multivariate OR } \\
\quad(95 \% \mathrm{CI})\end{array}$} & \multirow[t]{2}{*}{$p$-Value } \\
\hline & $N$ & $\%$ & & & & \\
\hline \multicolumn{7}{|l|}{ Sex: } \\
\hline Male & $211 / 241$ & 87.55 & 1.00 (reference) & & 1.00 (reference) & \\
\hline Female & $106 / 122$ & 86.89 & $0.94(0.49-1.80)$ & 0.857 & $0.76(0.38-1.54)$ & 0.451 \\
\hline \multicolumn{7}{|l|}{ Age at TST in Years: } \\
\hline $15-24$ & $88 / 97$ & 90.72 & 1.00 (reference) & & 1.00 (reference) & \\
\hline $25-34$ & $107 / 127$ & 84.25 & $0.55(0.24-1.26)$ & 0.157 & $0.54(0.22-1.31)$ & 0.173 \\
\hline $35-44$ & $80 / 93$ & 86.02 & $0.63(0.26-1.55)$ & 0.314 & $0.57(0.21-1.56)$ & 0.273 \\
\hline$\geq 45$ & $42 / 46$ & 91.30 & $1.07(0.31-3.69)$ & 0.910 & $1.39(0.33-5.86)$ & 0.658 \\
\hline Continuous OR & & & $0.98(0.72-1.34)$ & 0.892 & $1.01(0.70-1.45)$ & 0.956 \\
\hline \multicolumn{7}{|l|}{ Region of Origin: } \\
\hline Africa & $97 / 109$ & 88.99 & 1.00 (reference) & & 1.00 (reference) & \\
\hline Eastern Mediter. & $73 / 83$ & 87.95 & $0.90(0.37-2.20)$ & 0.823 & $0.93(0.35-2.47)$ & 0.892 \\
\hline Europe & $60 / 72$ & 83.33 & $0.62(0.26-1.47)$ & 0.275 & $0.72(0.27-1.91)$ & 0.509 \\
\hline SE Asia/West Pacific & $78 / 87$ & 89.66 & $1.07(0.43-2.67)$ & 0.881 & $2.39(0.72-7.93)$ & 0.155 \\
\hline Americas & $9 / 12$ & 75.00 & $0.37(0.09-1.56)$ & 0.177 & $0.32(0.06-1.64)$ & 0.174 \\
\hline TB Incidence $^{\circ}$ : & & & $1.11(0.82-1.52)$ & 0.489 & $0.99(0.70-1.40)$ & 0.975 \\
\hline \multicolumn{7}{|l|}{ Any Symptoms *: } \\
\hline No & $203 / 235$ & 86.38 & 1.00 (reference) & & & \\
\hline Yes & $114 / 128$ & 89.06 & $1.28(0.66-2.51)$ & 0.464 & & \\
\hline \multicolumn{7}{|l|}{ Cough: } \\
\hline No & $240 / 279$ & 86.02 & 1.00 (reference) & & 1.00 (reference) & \\
\hline Yes & $77 / 84$ & 91.67 & $1.79(0.77-4.16)$ & 0.178 & $2.38(0.92-6.17)$ & 0.073 \\
\hline Education **: & & & $1.09(0.73-1.63)$ & 0.660 & $1.09(0.71-1.68)$ & 0.688 \\
\hline Italian Language ${ }^{* * \circ}$ : & & & $1.13(0.68-1.87)$ & 0.636 & $1.15(0.67-1.95)$ & 0.616 \\
\hline \multicolumn{7}{|l|}{ Employment Status: } \\
\hline No & $228 / 257$ & 88.72 & 1.00 (reference) & & 1.00 (reference) & \\
\hline Yes & $81 / 98$ & 82.65 & $0.61(0.32-1.16)$ & 0.131 & $0.56(0.25-1.26)$ & 0.162 \\
\hline \multicolumn{7}{|l|}{ Years in Italy: } \\
\hline$<5$ & $215 / 249$ & 86.35 & 1.00 (reference) & & 1.00 (reference) & \\
\hline$\geq 5$ & $95 / 107$ & 88.79 & $1.25(0.62-2.52)$ & 0.530 & $1.88(0.80-4.40)$ & 0.145 \\
\hline Continuous OR & & & $1.04(0.95-1.13)$ & 0.376 & $0.99(0.64-1.54)$ & 0.972 \\
\hline \multicolumn{7}{|l|}{ Homelessness: } \\
\hline No & $299 / 340$ & 87.94 & 1.00 (reference) & & 1.00 (reference) & \\
\hline Yes & $18 / 23$ & 78.26 & $0.49(0.17-1.40)$ & 0.185 & $0.35(0.11-1.12)$ & 0.076 \\
\hline \multicolumn{7}{|l|}{ Refugees: } \\
\hline No & $290 / 335$ & 86.57 & 1.00 (reference) & & & \\
\hline Yes & $27 / 28$ & 96.43 & $4.19(0.56-31.60)$ & 0.165 & & \\
\hline \multicolumn{7}{|l|}{ Prostitution: } \\
\hline No & $309 / 352$ & 87.78 & 1.00 (reference) & & & \\
\hline Yes & $8 / 11$ & 72.73 & $0.37(0.09-1.45)$ & 0.155 & & \\
\hline \multicolumn{7}{|l|}{ Pregnancy: } \\
\hline No & $309 / 355$ & 87.04 & - & & & \\
\hline Yes & $8 / 8$ & 100.00 & - & $0.277^{* * *}$ & & \\
\hline \multicolumn{7}{|l|}{ TST result: } \\
\hline Negative & $210 / 210$ & 100.00 & - & & & \\
\hline Positive & $107 / 134$ & 79.85 & - & $0.000^{* * *}$ & & \\
\hline
\end{tabular}

${ }^{\circ}:$ TB incidence in the country of origin is sub-divided into four categories: 0-49, 50-99, 100-199, $\geq 200$ per 100,000 population/year. *: Meaning at least one among cough, hemoptysis, fever, chest pain, weight loss, fatigue, night sweats, chills or loss of appetite. ${ }^{* *}$ : Education is sub-divided into three categories: illiterate/primary school, secondary school, high school/degree. ${ }^{* * \circ}$ : Knowledge of Italian is sub-divided into three categories: none, sufficient, good. ***: Two-sample Mann-Whitney test. TB: tuberculosis; LTBI: latent tuberculosis infection; TST: tuberculin skin test; SE Asia: South-East Asia. 
Table 4. Association between selected characteristics and compliance with the full protocol (bivariate and multivariate analysis).

\begin{tabular}{|c|c|c|c|c|c|c|}
\hline \multicolumn{3}{|c|}{ Protocol Started/Completed } & \multirow{2}{*}{$\begin{array}{c}\text { Bivariate OR } \\
\quad(95 \% \mathrm{CI})\end{array}$} & \multirow{2}{*}{$p$-Value } & \multirow{2}{*}{$\begin{array}{l}\text { Multivariate } \\
\text { OR }(95 \% \text { CI) } \\
\end{array}$} & \multirow{2}{*}{$p$-Value } \\
\hline Sample characteristics & $N$ & $\%$ & & & & \\
\hline \multicolumn{7}{|l|}{ Sex: } \\
\hline Male & $183 / 243$ & 75.31 & 1.00 (reference) & & 1.00 (reference) & \\
\hline Female & $98 / 125$ & 78.40 & $1.19(0.71-1.99)$ & 0.509 & $1.18(0.65-2.12)$ & 0.588 \\
\hline \multicolumn{7}{|l|}{ Age at TST in Years: } \\
\hline $15-24$ & $83 / 97$ & 85.57 & 1.00 (reference) & & 1.00 (reference) & \\
\hline $25-34$ & $97 / 130$ & 74.62 & $0.50(0.25-0.99)$ & 0.046 & $0.44(0.21-0.93)$ & 0.031 \\
\hline $35-44$ & $70 / 95$ & 73.68 & $0.47(0.23-0.98)$ & 0.043 & $0.39(0.17-0.88)$ & 0.024 \\
\hline$\geq 45$ & $31 / 46$ & 67.39 & $0.35(0.15-0.81)$ & 0.014 & $0.26(0.10-0.68)$ & 0.006 \\
\hline Continuous OR & & & $0.74(0.58-0.94)$ & 0.014 & $0.67(0.50-0.89)$ & 0.007 \\
\hline \multicolumn{7}{|l|}{ Region of Origin: } \\
\hline Africa & $85 / 111$ & 76.58 & 1.00 (reference) & & 1.00 (reference) & \\
\hline Eastern Mediterranean & $62 / 83$ & 74.70 & $0.90(0.47-1.75)$ & 0.763 & $0.87(0.41-1.85)$ & 0.723 \\
\hline Europe & $49 / 72$ & 68.06 & $0.65(0.34-1.26)$ & 0.205 & $0.90(0.42-1.97)$ & 0.801 \\
\hline SE Asia/West Pacific & $76 / 90$ & 84.44 & $1.66(0.81-3.41)$ & 0.167 & $4.48(1.57-12.73)$ & 0.005 \\
\hline Americas & $9 / 12$ & 75.00 & $0.92(0.23-3.64)$ & 0.903 & $0.67(0.14-3.16)$ & 0.617 \\
\hline TB Incidence $^{\circ}$ : & & & $0.91(0.72-1.16)$ & 0.461 & $0.79(0.59-1.05)$ & 0.107 \\
\hline \multicolumn{7}{|l|}{ Any Symptoms *: } \\
\hline No & $179 / 236$ & 75.85 & 1.00 (reference) & & & \\
\hline Yes & $102 / 132$ & 77.27 & $1.08(0.65-1.79)$ & 0.758 & & \\
\hline \multicolumn{7}{|l|}{ Cough: } \\
\hline No & $212 / 280$ & 75.71 & 1.00 (reference) & & 1.00 (reference) & \\
\hline Yes & $69 / 88$ & 78.41 & $1.16(0.65-2.07)$ & 0.604 & $1.75(0.88-3.46)$ & 0.110 \\
\hline Education **: & & & $1.18(0.86-1.62)$ & 0.306 & $1.24(0.88-1.74)$ & 0.220 \\
\hline Italian Language ${ }^{* * \circ}$ : & & & $1.26(0.85-1.89)$ & 0.254 & $1.30(0.82-2.04)$ & 0.265 \\
\hline \multicolumn{7}{|l|}{ Employment Status: } \\
\hline No & $201 / 258$ & 77.91 & 1.00 (reference) & & 1.00 (reference) & \\
\hline Yes & $75 / 102$ & 73.53 & $0.79(0.46-1.34)$ & 0.377 & $0.59(0.30-1.17)$ & 0.133 \\
\hline \multicolumn{7}{|l|}{ Years in Italy: } \\
\hline$<5$ & $188 / 252$ & 74.60 & 1.00 (reference) & & 1.00 (reference) & \\
\hline$\geq 5$ & $88 / 109$ & 80.73 & $1.43(0.82-2.48)$ & 0.209 & $2.94(1.44-6.01)$ & 0.003 \\
\hline Continuous OR & & & $1.06(0.99-1.13)$ & 0.119 & $1.30(0.90-1.86)$ & 0.160 \\
\hline \multicolumn{7}{|l|}{ Homelessness: } \\
\hline No & $267 / 345$ & 77.39 & 1.00 (reference) & & 1.00 (reference) & \\
\hline Yes & $14 / 23$ & 60.87 & $0.45(0.19-1.09)$ & 0.077 & $0.35(0.13-0.96)$ & 0.042 \\
\hline \multicolumn{7}{|l|}{ Refugees: } \\
\hline No & $258 / 340$ & 75.88 & 1.00 (reference) & & & \\
\hline Yes & $23 / 28$ & 82.14 & $1.46(0.54-3.97)$ & 0.456 & & \\
\hline \multicolumn{7}{|l|}{ Prostitution: } \\
\hline No & $273 / 357$ & 76.47 & 1.00 (reference) & & & \\
\hline Yes & $8 / 11$ & 72.73 & $0.82(0.21-3.16)$ & 0.774 & & \\
\hline \multicolumn{7}{|l|}{ Pregnancy: } \\
\hline No & $272 / 359$ & 75.84 & - & & & \\
\hline Yes & $9 / 9$ & 91.67 & - & $0.091^{* * *}$ & & \\
\hline \multicolumn{7}{|l|}{ TST Result: } \\
\hline Negative & $210 / 210$ & 100.00 & - & & & \\
\hline Positive & $71 / 139$ & 51.08 & - & $0.000^{* * *}$ & & \\
\hline
\end{tabular}

${ }^{\circ}$ TB incidence in the country of origin is sub-divided into 4 categories: 0-49, 50-99, 100-199, $\geq 200$ per 100,000 population/year. ${ }^{*}$ Meaning at least one among cough, hemoptysis, fever, chest pain, weight loss, fatigue, night sweats, chills or loss of appetite. ** Education is sub-divided into three categories: illiterate/primary school, secondary school, high school/degree. ${ }^{* *}$ Knowledge of Italian is sub-divided into three categories: none, sufficient, good. ${ }^{* * *}$ Two-sample Mann-Whitney test. TST: tuberculin skin test; SE Asia: South-East Asia; TB: tuberculosis. 


\section{Discussion}

In our study, adherence with the screening of active TB and LTBI and with pulmonary TB therapy was very high compared to other recent investigations conducted in the USA, Switzerland, and Italy [13-17]. However, a direct comparison between these studies is difficult because study design, countries of origin and sample size were different.

In our study, we identified few fragile groups on which to focus to improve the performance of the screening program [18]. In particular, immigrants from America, Europe, Nigeria, Tunisia, and Georgia showed very low rates of compliance with screening, highlighting the need to target these subgroups with tailored procedures. Immigrants who were employed had lower overall compliance, probably due to the absence of health protection plans and the difficulties in acquiring health permits for these irregular workers. Conversely, knowledge of the Italian language was not significantly associated with compliance. The systematic presence of cultural mediators may have played a key role in determining this outcome, leading the immigrants to fully understand the significance of the screening program and the treatments they underwent, and to freely express questions and concerns $[9,19,20]$.

Our study has a few strengths. First of all, the decision to include in the study the whole population of illegal immigrants should be mentioned. Had we not included eligible immigrants from countries with low TB incidence, we would have excluded about $48 \%$ of the subjects and missed the $80 \%$ of TB cases. Therefore the model adopted in our centre is innovative because it is based on the presence of a stable team of specialists and a nurse dedicated to the periodic monitoring of compliance and to the recovery of the subjects that don't show up for appointments. Finally, the fact that all the investigations were for free and that there was a direct delivery of the therapy are important elements in ensuring such a high adherence to screening and treatment.

Our study also has limitation. To begin with, the immigrants' self-reported data on education, employment status and symptoms are likely to imply an information bias that may have limited the accuracy of such data. Furthermore, we have not studied the correlation between adherence to screening and characteristics such drinking habits and drug abuse, smoking, or possible congenital or acquired immunodeficiency disorders. Moreover, the small size of the study population led to a lack of statistical precision in our results, which need to be confirmed in larger populations. In addition, in patients with negative TST, QFT was not systematically performed and as reported in recent studies [21], QFT might increase the identification of LTBI cases in recent immigrants. Finally, the findings of this study and the investigated setting may not be easily extended to other contexts, since the model adopted in the study centre was based on the fact that all procedures were for free, and were characterized by a stable health care team. This team also included a nurse dedicated to the periodic monitoring of compliance and to making contact with subjects who failed to show up for appointments.

\section{Conclusions}

In conclusion, irregular immigrants appear to be a population with a high prevalence of LTBI and active TB and risk of progression from LTBI to active TB, being also characterized by delays in diagnosis and impaired referral to the health services. Consequently, they should be actively targeted with appropriate screening and follow-up procedures, particularly for selected subgroups showing lower compliance with screening and follow-up. Because of the high probability of diagnostic delay and poor accessibility to health services, systematic active TB screening should be implemented in each clinic dealing with seeking care irregular immigrants. In addition, the simultaneous execution of both the TB and LTBI screenings may optimize allocation and use of the resources.

Supplementary Materials: The following are available online at http:/ /www.mdpi.com/1660-4601/16/1/28/s1, Table S1: Demographic characteristics of the study population. Table S2: Association between countries of origin, sex and compliance with screening for active tuberculosis (TB), screening for latent tuberculosis infection (LTBI) and the full protocol (multivariate analysis). Table S3: Association between selected characteristics and compliance with Tuberculin Skin Test (TST) reading (bivariate and multivariate analysis). Table S4: Association between selected characteristics and compliance with chest-X-ray (CXR) execution (bivariate and multivariate analysis). 
Table S5: Association between selected characteristics and compliance with Quantiferon-TB gold (QFT) execution (bivariate and multivariate analysis). Table S6: Association between selected characteristics and outcome of Latent Tuberculosis Infection (LTBI) treatment (bivariate analysis).

Author Contributions: Conceptualization, B.F., C.S. and V.M.; Methodology, B.F., C.S., F.R. and V.M.; Software and Formal Analysis, B.F., C.S. and V.M.; Validation, V.M.; Investigation and Resources, B.F., F.R., C.C., G.M., M.L., M.C. and N.F.; Data Curation, B.F., C.S. and V.M.; Writing-original draft preparation, B.F., C.S., and V.M.; Writing-Review and Editing, B.F., C.S., F.R., C.C., M.C., G.M., M.L., N.F., and V.M.; Visualization, B.F., C.S., V.M.; Supervision and Project Administration, B.F. and V.M.; Funding Acquisition, V.M.

Funding: Funding provided by the Local Health Unit of Reggio Emilia.

Acknowledgments: In this section you can acknowledge any support given which is not covered by the author contribution or funding sections. This may include administrative and technical support, or donations in kind (e.g., materials used for experiments).

Conflicts of Interest: The authors declare no conflict of interest.

\section{References}

1. Floyd, K.; Glaziou, P.; Zumla, A.; Raviglione, M. The global tuberculosis epidemic and progress in care, prevention, and research: An overview in year 3 of the End TB era. Lancet Respir. Med. 2018, 6, $299-314$. [CrossRef]

2. WHO. Global Tuberculosis Report 2016; WHO: Geneva, Switzerland, 2016.

3. Matteelli, A.; Rendon, A.; Tiberi, S.; Al-Abri, S.; Voniatis, C.; Carvalho, A.C.C.; Centis, R.; D'Ambrosio, L.; Visca, D.; Spanevello, A.; et al. Tuberculosis elimination: Where are we now? Eur. Respir. Rev. 2018, 27. [CrossRef] [PubMed]

4. Lonnroth, K.; Migliori, G.B.; Abubakar, I.; D'Ambrosio, L.; de Vries, G.; Diel, R.; Douglas, P.; Falzon, D.; Gaudreau, M.A.; Goletti, D.; et al. Towards tuberculosis elimination: An action framework for low-incidence countries. Eur. Respir. J. 2015, 45, 928-952. [CrossRef] [PubMed]

5. Sanchez-Montalva, A.; Salvador, F.; Molina-Morant, D.; Molina, I. Tuberculosis and immigration. Enfermedades Infecciosas y Microbiologia Clinica 2018, 36, 446-455. [CrossRef] [PubMed]

6. WHO. The End TB Strategies; WHO: Geneva, Switzerland, 2014.

7. Kaushik, N.; Lowbridge, C.; Scandurra, G.; Dobler, C.C. Post-migration follow-up programme for migrants at increased risk of developing tuberculosis: A cohort study. ERJ Open Res. 2018, 4. [CrossRef] [PubMed]

8. IsdseAsesdRE-R. La Tubercolosi in Italia, Rapporto 2008; Ministero della Salute: Roma, Italy, 2010.

9. Salute, M.D. Aggiornamento Delle Raccomandazioni per le Attività di Controllo Della Tubercolosi. Gestione dei Contatti e Della Tubercolosi in Ambito Assistenziale; Istituto Nazionale Malattie Infettive Lazzaro Spallanzani IRCCS: Roma, Italy, 2010.

10. Salute, M.D. Aggiornamento Delle Raccomandazioni per le Attività di Controllo Della Tubercolosi: Politiche Efficaci a Contrastare la Tubercolosi Nella Popolazione Immigrata; Ministero della Salute: Roma, Italy, 2010.

11. CDC. Targeted Tubercolin Testing and Treatment of Latent Tubercolosis Inferction; CDC: Atlanta, GA, USA, 2000.

12. WHO. Definitions and Reporting Framework for Tuberculosis-2013 Revision; WHO: Geneva, Switzerland, 2014.

13. Morano, J.P.; Walton, M.R.; Zelenev, A.; Bruce, R.D.; Altice, F.L. Latent tuberculosis infection: Screening and treatment in an urban setting. J. Community Health 2013, 38, 941-950. [CrossRef] [PubMed]

14. Bennett, R.J.; Brodine, S.; Waalen, J.; Moser, K.; Rodwell, T.C. Prevalence and treatment of latent tuberculosis infection among newly arrived refugees in San Diego County, January 2010-October 2012. Am. J. Public Health 2014, 104, e95-e102. [CrossRef] [PubMed]

15. Bodenmann, P.; Vaucher, P.; Wolff, H.; Favrat, B.; de Tribolet, F.; Masserey, E.; Zellweger, J.P. Screening for latent tuberculosis infection among undocumented immigrants in Swiss healthcare centres; a descriptive exploratory study. BMC Infect. Dis. 2009, 9, 34. [CrossRef] [PubMed]

16. El-Hamad, I.; Casalini, C.; Matteelli, A.; Casari, S.; Bugiani, M.; Caputo, M.; Bombana, E.; Scolari, C.; Moioli, R.; Scarcella, C.; et al. Screening for tuberculosis and latent tuberculosis infection among undocumented immigrants at an unspecialised health service unit. Int. J. Tuberc. Lung Dis. 2001, 5, 712-716. [PubMed]

17. Schepisi, M.S.; Gualano, G.; Piselli, P.; Mazza, M.; D'Angelo, D.; Fasciani, F.; Barbieri, A.; Rocca, G.; Gnolfo, F.; Olivani, P.; et al. Active Tuberculosis Case Finding Interventions Among Immigrants, Refugees and Asylum Seekers in Italy. Infect. Dis. Rep. 2016, 8, 6594. [CrossRef] [PubMed] 
18. WHO. Guidelines on the Management of Latent Tuberculosis Infection; WHO: Geneva, Switzerland, 2015.

19. Suphanchaimat, R.; Kantamaturapoj, K.; Putthasri, W.; Prakongsai, P. Challenges in the provision of healthcare services for migrants: A systematic review through providers' lens. BMC Health Serv. Res. 2015, 15, 390. [CrossRef] [PubMed]

20. Cheng, I.H.; Drillich, A.; Schattner, P. Refugee experiences of general practice in countries of resettlement: A literature review. Br. J. Gen. Pract. 2015, 65, e171-e176. [CrossRef] [PubMed]

21. Saracino, A.; Scotto, G.; Fornabaio, C.; Martinelli, D.; Faleo, G.; Cibelli, D.; Tartaglia, A.; Di Tullio, R.; Fazio, V.; Prato, R.; et al. QuantiFERON-TB Gold In-Tube test (QFT-GIT) for the screening of latent tuberculosis in recent immigrants to Italy. New Microbiol. 2009, 32, 369-376. [PubMed]

C 2018 by the authors. Licensee MDPI, Basel, Switzerland. This article is an open access article distributed under the terms and conditions of the Creative Commons Attribution (CC BY) license (http://creativecommons.org/licenses/by/4.0/). 